\title{
A New Approach to Estimate the Complex Relative Permittivity of Materials Based on Free-Space Reflection Method
}

\author{
Le Cuong Nguyen ${ }^{1}$, Manh Cuong Ho ${ }^{1 *}$, Trong-Hieu Le ${ }^{1,2}$ \\ ${ }^{1}$ Faculty of Electronics and Telecommunications, Electric Power University, Hanoi, Vietnam \\ ${ }^{2}$ State Key Laboratory of Millimeter Waves, Southeast University, Nanjing, P. R. China \\ *Corresponding author: Manh Cuong Ho, E-mail: cuonghmeepu.edu.vn
}

\begin{abstract}
A microwave free-space reflection method for determining the complex permittivity of planar materials is demonstrated. The improved the MUltiple SIgnal Classification (MUSIC) algorithm is used to estimate the complex relative permittivity of material based on the reflection coefficients measurement of a metal-backed material sample placed in a free-space with the thickness of the samples is the much greater than the wavelength. The measurement system consists of a pyramidal horn antenna operating at X-band and a metal-backed sample placed in a free-space. From the measured values of the reflection coefficients through a known thickness of a planar slab of the material sample, the complex relative permittivity of the material samples is estimated by the proposed algorithm without determining an integer time the wavelength in the sample under test. The proposed algorithm is verified with different thickness material samples having different thicknesses at X-band. The estimation results show that the complex relative permittivity of material samples is accurate when thickness changes.
\end{abstract}

\section{Introduction}

Permittivity is a physical quantity that describes how a dielectric material responds to an electric field. The knowledge of permittivity is of great importance in various areas of science and engineering. Several methods to measure the complex permittivity of dielectric materials have been developed. Among these methods, the methods for determining the parameters of material using microwave propagation in free-space are nondestructive, contactless, and sample preparation requirements are minimal. Therefore, they are especially suitable for measurement of the complex permittivity and complex permeability of materials under high-temperature conditions [1], [2]. For these reasons, they can be implemented in industrial processes where particular parameters such as density and moisture content need to be monitored in real time [3]-[5]. The free-space methods are based on the measurements of the phase of the reflection $\left(\mathrm{S}_{11}\right)$ and transmission $\left(\mathrm{S}_{21}\right)$ coefficient through a known thickness of the material samples. The reflection and transmission coefficients are determined by measuring the attenuation and phase shift introduced by a sample placed between two antennas. However, when the wavelength in the material sample is smaller than the thickness of the material under test, a phase problem is encountered [6]-[13]. Because phase-angle measurements are only possible between -1800 and +1800 . The total phase shift is measured using the vector network analyzer, shifted by $\mathrm{n}$ times 3600 , where $\mathrm{n}$ is an integer to be determined. This problem was solved by making measurements on samples of different thickness [14] or by the delay time if the wave is non-dispersive in the observed frequency range [15] or by the measurement technique, based on reflection, for thickness and permittivity determination [16] or by the based on selecting a sample thickness that allows the phase to remain within the measurement limits of the instrumentation at a given frequency or two frequencies [17]. On the other hands, in our previous work [18], a proposed method to estimate the complex permittivity of the material samples placed in free space is carried out by a measurement system that consists of a transmitting and a receiving horn antennas to calculate transmission coefficient $\left(\mathrm{S}_{12}\right.$ or $\left.\mathrm{S}_{21}\right)$ electromagnetic wave propagation in free space.

In this paper, we propose a novel approach using a high-resolution algorithm based on the improved MUSIC algorithm [19] and exploiting the relationship of the permittivity and the refractive index of the material to solve the phase ambiguity problem. Different from [18], the proposed measurement system only consists of a pyramidal horn antenna operating at $8 \mathrm{GHz}$ to $12 \mathrm{GHz}$ frequency range and a metal-backed sample placed in a free-space. Therefore, the values of the reflection coefficients are measured through a known thickness of a planar slab material sample. Moreover, the proposed algorithm can be to estimate the complex relative permittivity of samples when the thickness is the much greater than the wavelength in the material sample without determining an integer time the wavelength in the sample under test.

The rest of this paper is organized as follows. Section 2 describes the reflection method while estimation procedure is presented in Section 3. Section 4 is the implementation modeling by Computer Simulation Tool (CST) and the simulated estimation results. Finally, a brief conclusion is given in Section 5. 


\section{Free-Space reflection method}

Fig.1 shows a typical setup for a free-space reflection measurement.

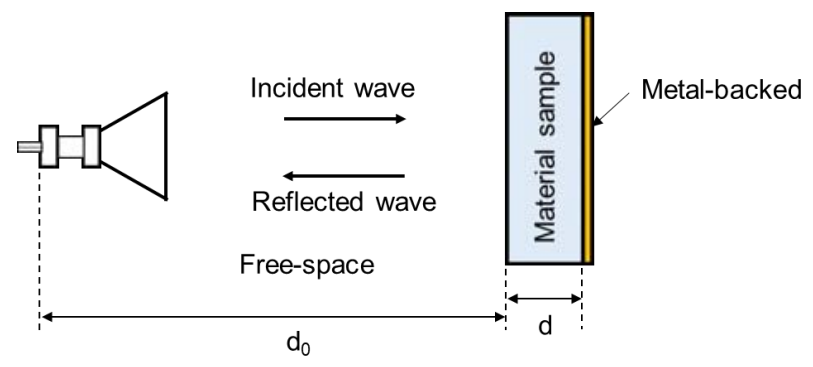

Figure 1: Free-space reflection method.

Reflection measurement of the system is only considered on $D$ discrete reflection points which are the frequency points from $f_{1}$ to $f_{\mathrm{M}}$ within the sampling frequency range (bandwidth equivalent to $f_{\mathrm{M}}-f_{1}$ ). If the number of multiple reflections is limited (and can be considered as negligible, then the number of signals is equal to $D$. The following formulation must also be applied to this measurement. It is assumed that the antenna has $D$ signal sources changing from $f_{1}$ to $f_{\mathrm{M}}$ and radiating through the free-space and material sample to the metal-back then reflected back. The data obtained by the system above are the reflection coefficients at $M$ uniformly sampled frequency points $f_{\mathrm{i}}(i=1,2, \ldots, M: M>D)$ with the same frequency $\Delta f$ between adjacent points. Delay time is represented corresponding to the $k^{\text {th }}$ reflection point by $t_{\mathrm{k}}$ ( $k=1,2, \ldots, D)$. Then, the measurement value of reflected signals at the frequency $f_{\mathrm{i}}$ is given by

$$
x_{\mathrm{i}}(\mathrm{t})=\sum_{k=1}^{D} s_{\mathrm{k}}(\mathrm{t}) \mathrm{e}^{-j 2 \pi f_{\mathrm{i}} t_{\mathrm{k}}}+w_{\mathrm{i}}(\mathrm{t})
$$

where $s_{\mathrm{k}}(\mathrm{t})$ is the reflection coefficient of the $k^{\text {th }}$ reflection point at the frequency $f_{\mathrm{i}}, w_{\mathrm{i}}(\mathrm{t})$ is the additive noise with zero mean and variance $\sigma^{2}$.

Fig. 1 shows a planar sample of thickness $d$ placed in free space. The complex permittivity, relative to free space, is defined as:

$$
\varepsilon^{*}=\varepsilon^{\prime}-j \varepsilon^{\prime \prime}
$$

where $\varepsilon^{\prime}$ and $\varepsilon^{\prime \prime}$ are the real and imaginary parts of the complex relative permittivity.

When the signal passes through the material sample, some part of it is always attenuated. To take this into account, an imaginary part is added to the refractive index. The complex refractive index $\left(\eta^{*}\right)$ is defined as:

$$
\eta^{*}=\eta^{\prime}-j \eta^{\prime \prime}
$$

where $\eta^{\prime}$ and $\eta^{\prime \prime}$ are the real and imaginary parts of the refractive index.

The complex relative permeability of non-magnetic materials is equal 1.0. The complex refractive index is related to the complex permittivity by the following equation:

$$
\eta^{*}=\sqrt{\varepsilon^{\prime}-j \varepsilon^{\prime \prime}}
$$

The time of arrival is also equal to delay time $\left(\tau=t_{\mathrm{k}}\right)$ and it is determined by

$$
\tau=2\left(d \frac{\sqrt{\varepsilon^{\prime}-j \varepsilon^{\prime \prime}}}{c}+d_{0} \frac{1}{c}\right)
$$

where $d_{0}$ is the distance between the port of antenna and material sample, $c$ is the light velocity in free-space.

The difference in phase $\left(\Delta \varphi_{\mathrm{i}}\right)$ of the signal when it goes through the medium (free space and material sample) at the first frequency and the $i^{\text {th }}$ frequency is:

$$
\Delta \varphi_{\mathrm{i}}=\frac{4 \pi(i-1) \Delta f}{c}\left(d \sqrt{\varepsilon^{\prime}-j \varepsilon^{\prime \prime}}+d_{0}\right)
$$

Combine this with the phase difference, the relation (1) can be written using the vector notation as follows:

$$
X=A S+W
$$

where:

$$
X=\left[x_{1}(\mathrm{t}), x_{2}(\mathrm{t}), \ldots x_{\mathrm{M}}(\mathrm{t})\right]^{T}, X \text { is the } M \mathrm{x} 1 \text { output }
$$
vector measured at receiver, $T$ denotes transpose.

$$
S=\left[s_{1}(\mathrm{t}), s_{2}(\mathrm{t}), \ldots, s_{\mathrm{D}}(\mathrm{t})\right]^{T}, S \text { is the vector of the } k
$$
arriving signals.

$$
W=\left[w_{1}(\mathrm{t}), w_{2}(\mathrm{t}), \ldots, w_{\mathrm{M}}(\mathrm{t})\right]^{T}, \quad W \text { is the noise }
$$

vector:

$A=\left[a\left(\varepsilon_{1}^{\prime}, \varepsilon_{1}^{\prime \prime}\right), a\left(\varepsilon_{2}^{\prime}, \varepsilon_{2}^{\prime \prime}\right), \ldots, a\left(\left(\varepsilon_{\mathrm{D}}^{\prime}, \varepsilon_{\mathrm{D}}^{\prime \prime}\right)\right]\right.$,
$M \mathrm{x} D$
"parameter" $a\left(\varepsilon_{\mathrm{k}}^{\prime}, \varepsilon_{\mathrm{k}}^{\prime \prime}\right)=\left[\mathrm{e}^{-j \Delta \varphi_{1}}, \mathrm{e}^{-j \Delta \varphi_{2}}, \ldots, \mathrm{e}^{-j \Delta \varphi_{\mathrm{i}}}\right]^{T}$ is a "parameter" vector of each signal.

\section{Complex relative permittivity estimation procedure}

The multiple signal classification algorithm was proposed by R. Schmidt [19]. The basic approach of this algorithm is that from the received signal, the covariance matrix is calculated and then eigenvectors decomposition is carried out. The signal subspace and noise subspace are determined based on eigenvectors and eigenvalues. The results showed that the $M$ - $D$ dimensional subspace spanned by the $M-D$ noise eigenvectors as the noise subspace and the $D$ dimensional subspace spanned by the incident signal parameter vectors as the signal subspace; they are disjoint. The signal and the 
noise subspaces are calculated by the matrix algebra and they are found to be orthogonal to each other. Therefore, the signal and noise subspaces are isolated by the orthogonal property of this algorithm. Thus, the complex relative permittivity of the material sample is estimated by combining the autocorrelation and MUSIC function of the received signal.

The corresponding data covariance matrix in (7) is given by

$$
R_{\mathrm{XX}}=E\left\{X X^{H}\right\}=A S_{\mathrm{XX}} A^{H}+\sigma^{2} I
$$

where $S_{\mathrm{XX}}=E\left\{S S^{H}\right\}$ denotes the signal covariance matrix, $I$ is the identity matrix, $H$ denotes complex conjugate transpose.

The eigenvalues of $R_{\mathrm{XX}}$ are $\lambda_{1}, \lambda_{2}, \ldots, \lambda_{\mathrm{D}}$ such that:

$$
\operatorname{det}\left(R_{\mathrm{Xx}}-\lambda_{\mathrm{i}} I\right)=0
$$

Substituting (8) to (9):

$$
\operatorname{det}\left(A S_{\mathrm{XX}} A^{H}-\left(\lambda_{\mathrm{i}}-\sigma^{2}\right) I\right)=0
$$

The eigenvalues $\xi_{\mathrm{i}}$ of $A S_{\mathrm{XX}} A^{H}$ are:

$$
\xi_{\mathrm{i}}=\lambda_{\mathrm{i}}-\sigma^{2}
$$

If the eigenvalues $\xi_{\mathrm{i}}$ of $A S_{\mathrm{XX}} A^{H}$ are zero, $A S_{\mathrm{XX}} A^{H}$ is singular. This means that the number of incident wave fronts $D$ is less than the number of frequency elements $M$. Thus, The minimum eigenvalue of $R_{\mathrm{XX}}$ is equivalent to $\sigma^{2}$ with multiplicity $M-D$. Therefore:

$$
\lambda_{1} \geq \lambda_{2} \geq \cdots \geq \lambda_{\mathrm{D}} \succ \lambda_{\mathrm{D}+1}=\lambda_{\mathrm{D}+2}=\cdots=\lambda_{\mathrm{M}}=\sigma^{2}
$$

The eigenvector $u_{\mathrm{i}}$ associated with the eigenvalue $\lambda_{\mathrm{i}}$ satisfies the following equation:

$$
\left(R_{\mathrm{XX}}-\lambda_{\mathrm{i}} I\right) u_{\mathrm{i}}=0
$$

For eigenvectors associated with the minimum eigenvalue, the (14) is suggested by substituting (8) and (12) into (13).

$$
A S_{\mathrm{XX}} A^{H} u_{\mathrm{i}}=0
$$

Since $A$ has full rank and $S_{X X}$ is non-singular, thus:

$$
A^{H} u_{\mathrm{i}}=0
$$

This means that the eigenvectors corresponding to the minimum eigenvalue are orthogonal to the columns of the matrix $A$. Namely, they are orthogonal to the "parameter" vector of the signals: $\left\{u_{\mathrm{D}+1}, \ldots, u_{\mathrm{M}}\right\} \perp\left\{a\left(\varepsilon_{1}^{\prime}, \varepsilon_{1}^{\prime \prime}\right), \ldots, a\left(\varepsilon_{\mathrm{D}}^{\prime}, \varepsilon_{\mathrm{D}}^{\prime \prime}\right)\right\}$.

It implies that the squared norm of $A^{H} u_{\mathrm{i}}$ is zero

$$
\left\|A^{H} u_{\mathrm{i}}\right\|^{2}=a^{H}\left(\varepsilon^{\prime}, \varepsilon^{\prime \prime}\right) U_{\mathrm{M}} U_{\mathrm{M}}^{H} a\left(\varepsilon^{\prime}, \varepsilon^{\prime \prime}\right)=0
$$

where $U_{\mathrm{M}}=\left[u_{\mathrm{D}+1}, u_{\mathrm{D}+2}, \ldots, u_{\mathrm{M}}\right]$ represents the eigenvectors associated with the noise subspace of the covariance matrix $R_{\mathrm{XX}}$.

The pseudo-spectrum of the MUSIC function as (17) is given by combining the autocorrelation function of signal subspace:

$$
P_{\text {MUSIC }}\left(\varepsilon^{\prime}, \varepsilon^{\prime \prime}\right)=\frac{a^{H}\left(\varepsilon^{\prime}, \varepsilon^{\prime \prime}\right) a\left(\varepsilon^{\prime}, \varepsilon^{\prime \prime}\right)}{a^{H}\left(\varepsilon^{\prime}, \varepsilon^{\prime \prime}\right) U_{\mathrm{M}} U_{\mathrm{M}}{ }^{H} a\left(\varepsilon^{\prime}, \varepsilon^{\prime \prime}\right)}
$$

The values of $\varepsilon^{\prime}$ and $\varepsilon^{\prime \prime}$ that make $P_{\text {Music }}$ reach a peak that are chosen from the result of the estimation.

\section{Implementation modeling and simulation results}

\subsection{System modeling}

In order to estimate the reflection coefficients $\left(S_{11}\right)$ for the free-space reflection method presented in section II, in this part, we have implemented the model using CST software, the flowchart and numerical setup performs modeling to determine parameter $S_{11}$ as shown in Figure 2.

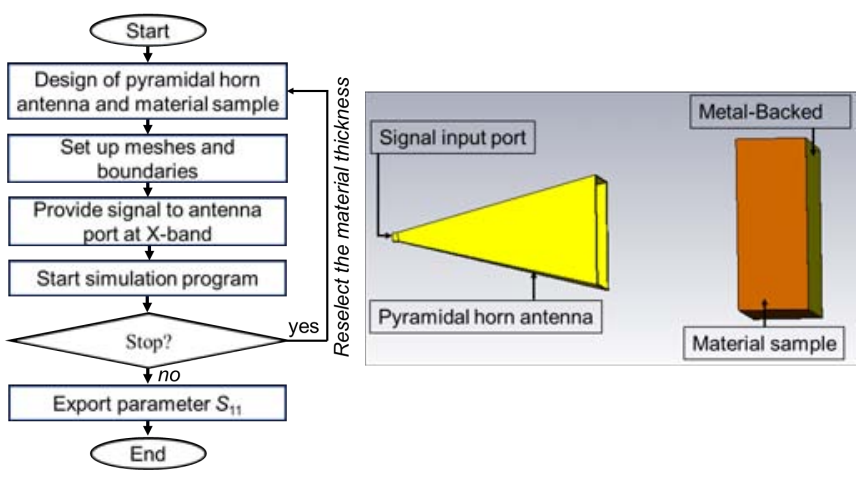

Figure 2: Modeling to determine parameter $S_{11}$ : Left (Flowchart) and Right (Numerical setup).

In Fig. 2, one pyramidal horn antenna is designed to operate well in the frequency range of $8.0-12.0 \mathrm{GHz}$ [20]. The gain and voltage standing wave ratio of the pyramidal horn antenna are $20 \mathrm{dBi}$ and 1.15 at center frequency. In this model, the distance between the port of antenna and the material sample is $1082.5 \mathrm{~mm}$. The calibration procedure requires to withdraw the losses due to free-space, this needing to proceed to the same measurements with air material. Table 1 shows the complex relative permittivity values of material samples at $10 \mathrm{GHz}$.

Table 1: The parameters of material samples.

\begin{tabular}{cccc}
\hline Material & $\varepsilon^{\prime}$ & $\varepsilon^{\prime \prime}$ & $\begin{array}{c}\text { Thickness } d \\
(\mathrm{~mm})\end{array}$ \\
\hline FR-4 (Lossy) & 4.3 & 0.025 & $10 \div 90$ \\
RO4350B (Lossy) & 3.66 & 0.0037 & $10 \div 90$ \\
$\begin{array}{c}\text { TEFLON-PTFE } \\
\text { (Lossy) }\end{array}$ & 2.1 & 0.0002 & $10 \div 90$ \\
\hline
\end{tabular}




\subsection{Simulation results}

The material samples are measured at 801 different frequencies from 8.0 to $12.0 \mathrm{GHz}$ with the scale of $5 \mathrm{MHz}$. The simulation was carried out on PCs with Intel Core i3 of memory chips, at $2.2 \mathrm{GHz}$ speed and $8 \mathrm{G}$ RAM. The total simulation and processing time for one example is about 2 hours' real time. From Fig. 3 to Fig. 7 show the pseudospectrum for the permittivity of FR-4 sample with thickness of $10 \mathrm{~mm}, 30 \mathrm{~mm}, 50 \mathrm{~mm}, 70 \mathrm{~mm}$ and $90 \mathrm{~mm}$, respectively.

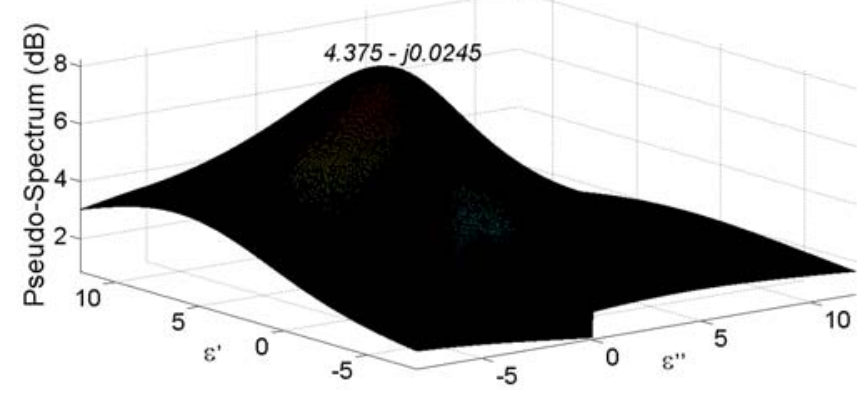

Figure 3: Pseudo-spectrum of FR-4 sample at 801 frequencies, frequency range $4.0 \mathrm{GHz}$ and thickness $10 \mathrm{~mm}$.

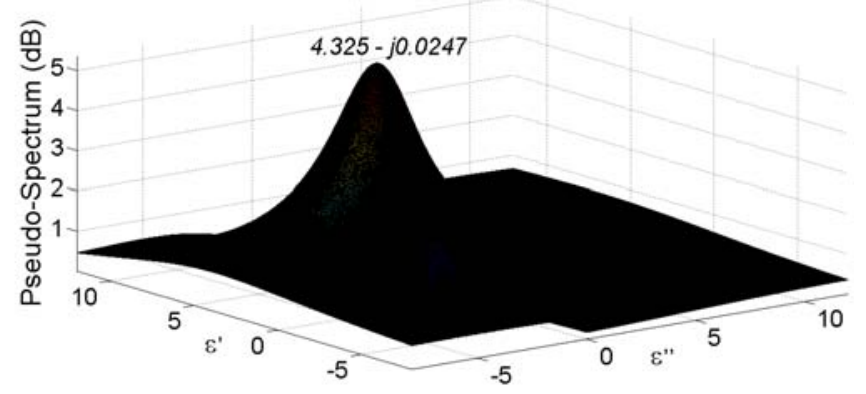

Figure 4: Pseudo-spectrum of FR-4 sample at 801 frequencies, frequency range $4.0 \mathrm{GHz}$ and thickness $30 \mathrm{~mm}$.

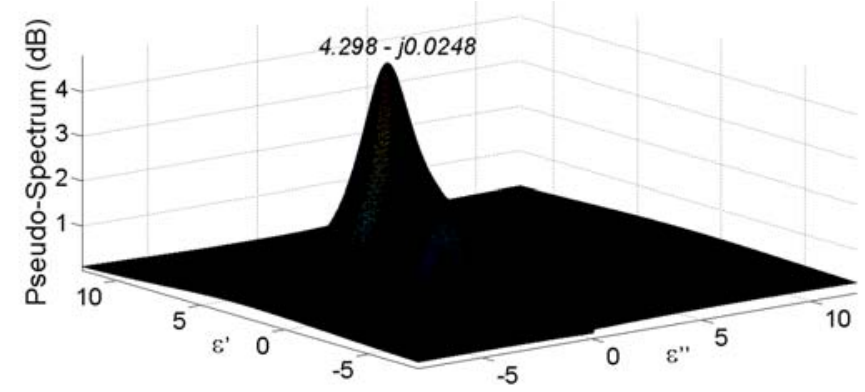

Figure 5: Pseudo-spectrum of FR-4 sample at 801 frequencies, frequency range $4.0 \mathrm{GHz}$ and thickness $50 \mathrm{~mm}$.

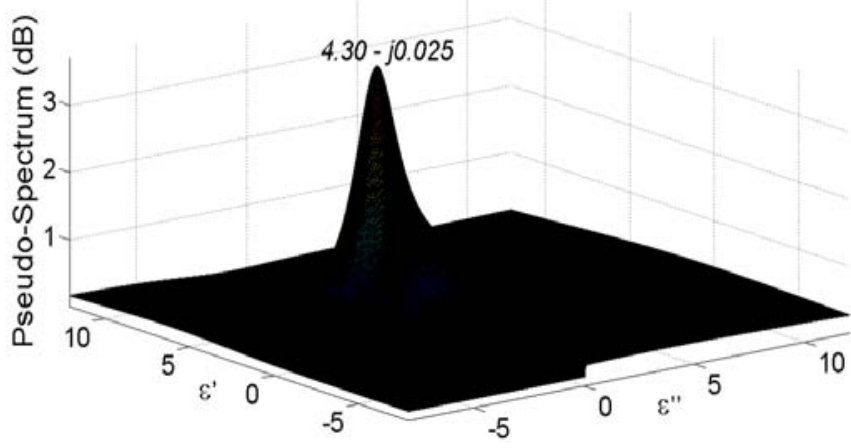

Figure 6: Pseudo-spectrum of FR-4 sample at 801 frequencies, frequency range $4.0 \mathrm{GHz}$ and thickness $70 \mathrm{~mm}$.

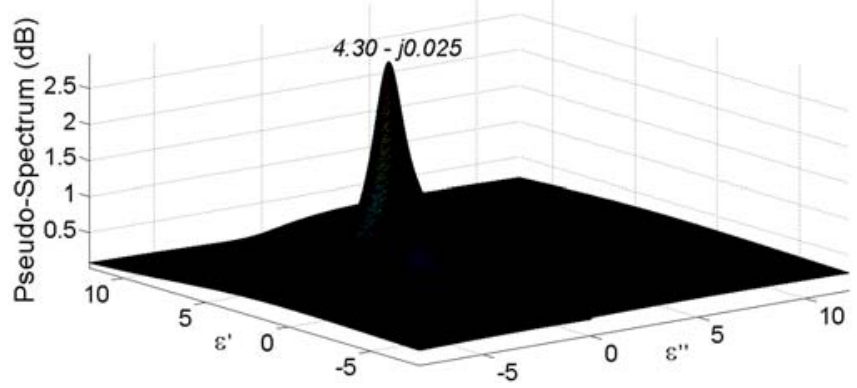

Figure 7: Pseudo-spectrum of FR-4 sample at 801 frequencies, frequency range $4.0 \mathrm{GHz}$ and thickness $90 \mathrm{~mm}$.

It can be seen that from Fig. 3, 4, 5, 6 and 7, respectively, the change in the thickness of the sample affects both the sharpness and the position of the peak of pseudo-spectrum. The change in the position of the peak from the expected value means that the estimation is not accurate for materials with small thickness. Furthermore, the drop in sharpness of the peak makes the determination of the point of maximum spectrum more difficult because the points around the peak can become equal to or greater than the supposed peak. These changes combined create a sharp decrease of the accuracy of the results as the thickness of the samples decreases.

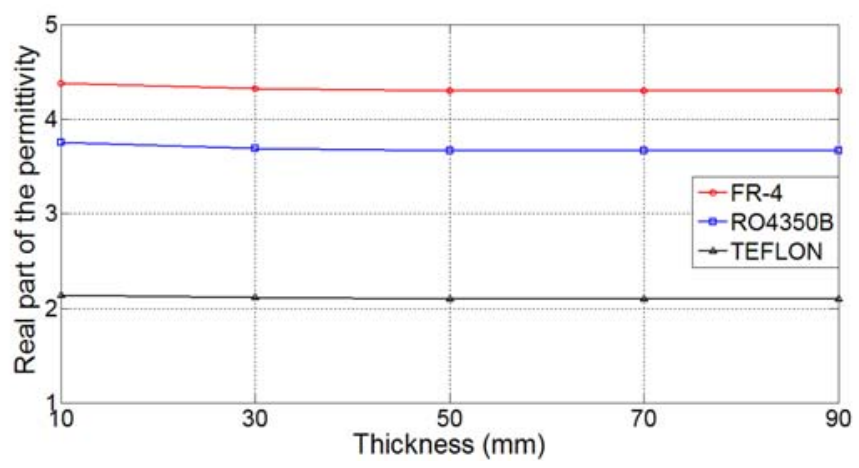

Figure 8: The real part of the complex relative permittivity of material samples is estimated by the MUSIC algorithm. 


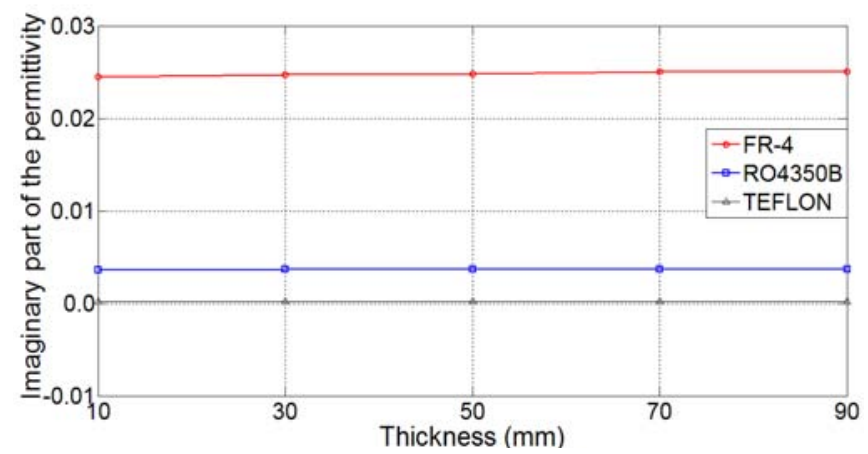

Figure 9: The imaginary part of the complex relative permittivity of material samples is estimated by the MUSIC algorithm.

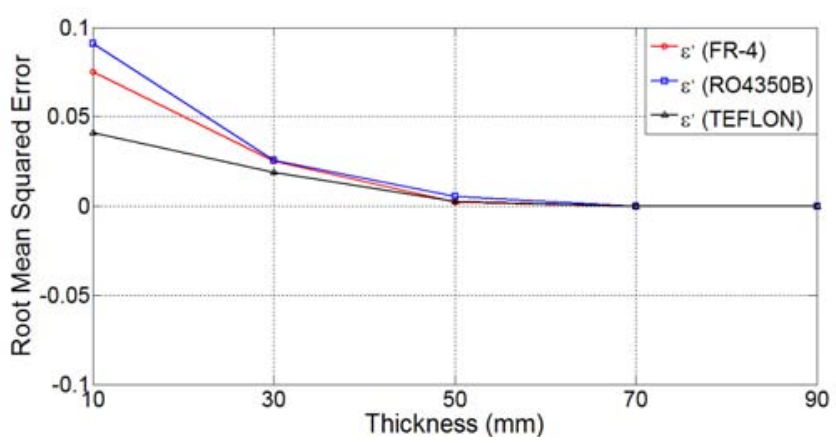

Figure 10: Root mean squared error versus thickness graph for $\varepsilon^{\prime}$.

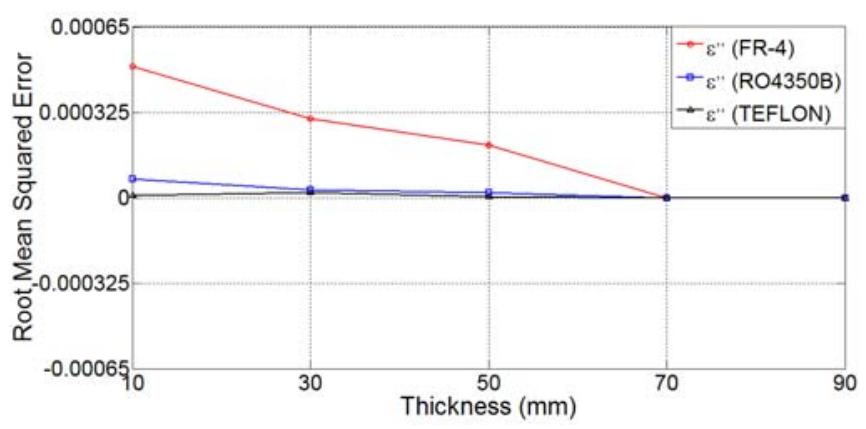

Figure 11: Root mean squared error versus thickness graph for $\varepsilon^{\prime \prime}$.

The estimation results show that the complex relative permittivity of material samples is accurately when thickness changes as Fig. 8 and Fig. 9, Fig. 10 and Fig. 11, respectively, show the root mean squared error (RMSE) versus the thickness graph calculated from the simulation results for the three samples at different thicknesses. From the results, we can see that the algorithm can solve for the unique value of the permittivity regardless of the thickness $\mathrm{d}$, but is more accurate with samples of larger thickness. The thickness of the sample affects the accuracy of the measurement of both $\varepsilon^{\prime}$ and $\varepsilon^{\prime \prime}$. To get the accurate value of $\varepsilon^{\prime}$, the thickness $d$ needs to be approximately $50 \mathrm{~mm}$ or higher, whereas it needs to be around $70 \mathrm{~mm}$ to get accurate results of both $\varepsilon^{\prime}$ and $\varepsilon^{\prime \prime}$.

\section{Conclusions}

A super high-resolution algorithm to estimate the complex relative permittivity of the planar material samples using free-space reflection method is proposed. The system model consists of a pyramidal horn antenna and a metal-backed material sample placed in a free-space. The parameter vectors of the MUSIC algorithm describe the difference in phase, which indicates the difference in frequencies and arrival time of the simulated signals. These parameter vectors are calculated by using the relation between the permittivity and the refractive index. The performance of the proposed algorithm is verified for all scenarios in the simulation. Through the results, the ability of the proposed algorithm to solve the problem of ambiguity of the conventional method is also validated. The measurement of the real part of the complex relative permittivity using this algorithm is accurately when the thickness of the material is at least $50 \mathrm{~mm}$. Whereas, the measurement of the imaginary part of the complex relative permittivity using this algorithm is accurate when the thickness of the material is at least $70 \mathrm{~mm}$. The proposed algorithm has great benefits for determining the characteristic parameters of new materials.

\section{Acknowledgements}

We would like to thank Faculty of Electronics and Telecommunications, Electric Power University (EPU), Hanoi, Vietnam for helping us in design-simulation facilities.

\section{References}

[1] D. K. Ghodgaonkar, V. V. Varadan, and V. K. Varadan, "A free-space method for measurement of dielectric constants and loss tangents at microwave frequencies," IEEE Transactions on Instrumentation and Measurement, vol. 38, pp. 789-793, 1989.

[2] Ghodgaonkar. D. K, Varadan. V. V, and Varadan. V. K, "Free-space measurement of complex permittivity and complex permeability of magnetic materials at microwave frequencies," Instrumentation and Measurement, IEEE Transactions on, vol. 39, pp. 387394, 1990.

[3] S. K. A. Kraszewski, and Z. Stosio, "A preliminary study on microwave monitoring of moisture content in wheat," J. Microw. Power, vol. 12, no.3, pp. 241-252, 1977.

[4] M. K. a. E. Kress-Rogers, "Microwave moisture and density measurements in particulate solids," Trans. Inst. Meas. Control, vol. 8, no3, pp. 161-168, 1986.

[5] S. Trabelsi, A. W. Kraszewski, and S. O. Nelson, "Simultaneous determination of density and water content of particulate materials by microwave sensors," Electronics Letters, vol. 33, pp. 874-876, 1997.

[6] E. Håkansson, A. Amiet, and A. Kaynak, "Electromagnetic shielding properties of polypyrrole/polyester composites in the $1-18 \mathrm{GHz}$ frequency range," Synthetic metals, vol. 156, pp. 917925, 2006. 
[7] V. V. Varadan and R. Ro, "Unique Retrieval of Complex Permittivity and Permeability of Dispersive Materials From Reflection and Transmitted Fields by Enforcing Causality," IEEE Transactions on Microwave Theory and Techniques, vol. 55, pp. 2224-2230, 2007.

[8] V. N. Semenenko and V. A. Chistyaev, "Measurement methods of complex permittivity and permeability of sheet samples in free space in microwave range," In 20th International Crimean Conference Microwave \& Telecommunication Technology, pp. 1091-1092, 2010.

[9] J. Roelvink and S. Trabelsi, "Measuring the complex permittivity of thin grain samples by the free-space transmission technique," In Instrumentation and Measurement Technology Conference (I2MTC), IEEE International, pp. 310-313, 2012.

[10]R. A. Fenner and S. Keilson, "Free space material characterization using genetic algorithms," In Antenna Technology and Applied Electromagnetics (ANTEM), 2014 16th International Symposium on, pp. 1-2, 2014.

[11] K. Haddadi and T. Lasri, "Geometrical Optics-Based Model for Dielectric Constant and Loss Tangent FreeSpace Measurement," IEEE Transactions on Instrumentation and Measurement, vol. 63, pp. 1818$1823,2014$.

[12] N. A. Andrushchak, I. D. Karbovnyk, K. Godziszewski, Y. Yashchyshyn, M. V. Lobur, and A. S. Andrushchak, "New Interference Technique for Determination of Low Loss Material Permittivity in the Extremely High Frequency Range," IEEE Transactions on Instrumentation and Measurement, vol. 64, pp. 3005$3012,2015$.

[13] T. Tosaka, K. Fujii, K. Fukunaga, and A. Kasamatsu, "Development of Complex Relative Permittivity Measurement System Based on Free-Space in 220-330 GHz Range," IEEE Transactions on Terahertz Science and Technology, vol. 5, pp. 102-109, 2015.

[14]H. Altschuler, "Dielectric constant," In Handbook of Microwave Measurements, M. Sucher and J. Fox, Eds. Brooklyn, NY: Polytechnic Press, Vol. 3, 1963.

[15] A. Klein, "Microwave moisture determination of coal A comparison of attenuation and phase measurement," In Proc. 10th Euro. Microwave Conf., vol. 1, pp. 526530, 1980.

[16]P. J. Joseph, J. C. Joseph, D. P. Glynn, III, and T.D. Perkins, III, "A portable vector reflectometer and its application for thickness and permittivity measurements," Microwave J., vol. 2, no. 12, pp. 8490, 1994.

[17] S. Trabelsi, A.W. Kraszewski, and S. O. Nelson, "Phase-shift ambiguity in microwave dielectric properties measurements," IEEE Transactions on Instrumentation and Measurement, vol. 49, pp. 56-60, 2000.

[18]H. Manh Cuong, N. Trong Duc and V. Van Yem, "Measurement of Complex Permittivity of Materials Using Electromagnetic Wave Propagation in Free Space and Super High-Resolution Algorithm," 2017 International Conference on Advanced Technologies for Communications (ATC), pp. 156-160, 2017.
[19] R.O. Schmidt, "Multiple emitter location and signal parameter estimation", IEEE Transactions on Antennas and Propagation, vol. 34, pp. 276-280, 1986.

[20]I. P. Arvind Roy, "Design and Analysis of X band Pyramidal Horn Antenna Using HFSS", International Journal of Advanced Research in Electronics and Communication Engineering, vol. 4, pp. 488-493, 2015. 